\title{
Using Predictable Observer Mobility for Power Efficient Design of Sensor Networks
}

\author{
Arnab Chakrabarti, Ashutosh Sabharwal, and Behnaam Aazhang \\ Rice University, Houston TX 77005, USA
}

\begin{abstract}
In this paper, we explore a novel avenue of saving power in sensor networks based on predictable mobility of the observer (or data sink). Predictable mobility is a good model for public transportation vehicles (buses, shuttles and trains), which can act as mobile observers in wide area sensor networks. To understand the gains due to predictable mobility, we model the data collection process as a queuing system, where random arrivals model randomness in the spatial distribution of sensors. Using the queuing model, we analyze the success in data collection, and quantify the power consumption of the network. Even though the modeling is performed for a network which uses only single hop communication, we show that the power savings over a static sensor network are significant. Finally, we present a simple observer-driven communication protocol, which follows naturally from the problem formulation and can be used to achieve the predicted power savings.
\end{abstract}

\section{Introduction}

One of the major challenges in designing sensor networks is maximizing the useful network lifetime. Since many sensor networks deploy sensor nodes which are battery powered and which can possibly scavenge only a small amount of energy from their surroundings, limited battery is one of the major hurdles in achieving desired longevity of network operation. Reducing power consumption in sensing and subsequent data collection has been a topic of extensive study [1$5]$. It has also been observed that communication power (which includes channel monitoring) is usually a significant component of the total power consumed in a sensor network $[1,6]$. In this paper, we explore the impact of predictable observer mobility in reducing communication power consumption in a sensor network.

Our contributions in this paper are two-fold. First, we propose a queuing formulation to accurately model data collection by the mobile observer over the region of interest. The queuing formulation captures the randomness due to random placement of sensors in the region. To achieve a pre-specified outage, defined as the fraction of nodes which fail to send their data, we show that predictability of the observer's motion can lead to large power savings over a network with no mobility. Second, we propose a simple observer-driven communication protocol which achieves a significant portion of the predicted gains. As the observer in our model is assumed to traverse the same path repeatedly, the data is pulled by the observer by waking up the nodes when it is close to them. Since the sensor 
nodes only transmit when the observer is close to them, the power requirements are significantly reduced.

Many strategies for reducing power consumption in sensor networks have been explored. In [6], many of the important avenues were identified, such as, increasing the number of sensors to reduce transmission range, reducing the standby power through suitable protocol design, and energy-efficient hardware implementation. Power efficient topologies for situations where sensor locations can be precisely specified were explored in [3]. In [7-9] some communication issues such as modulation, coding and multiple-access were studied in the context of sensor networks and power-saving solutions were proposed. In a different direction, it was shown in [10] that mobility of nodes can increase the capacity of ad hoc networks for applications with loose delay constraints. None of the above work, however, looked at the special case of exploiting predictable observer mobility to save power in a sensor network.

Our work is particularly motivated by the fact that a prototype of this proposed model is currently being built at Rice University where university shuttle buses will carry mobile observers and sensors will be deployed on buildings. Since these carriers, such as buses, usually have a source of power that is more than sufficient for communicating, storing and processing data, the observer is not power constrained like the sensor nodes. Furthermore, the shuttle buses have fixed and predictable routes.

The rest of the paper is organized as follows. In Section 2, we describe the proposed model. Section 3 explains the process of data collection by the mobile observer. In Section 4, we provide a power comparison between the proposed sensor network model and two static sensor network models. A protocol suitable for our paradigm is proposed in Section 5. Finally, we conclude in Section 6.

\section{Proposed Model}

The sensor network consists of $N$ sensor nodes distributed over an area $A$ (see Fig. 1). Two node distribution models are studied in this paper. In the first model, nodes are assumed to be independently and uniformly scattered over the area $A$. Random scattering is a good model for cases where cheap sensor nodes are dropped with no particular plan, for rapid deployment. In the second model, we assume that the sensors are placed such that no two sensors are less than $d$ meters apart, and the network is laid out to perform an efficient spatial sampling of the terrain.

The observer $O$ moves repeatedly along a deterministic route inside $A$. When the observer has moved over the entire area $A$ once and returned to the point from which it started, we say that it has completed a cycle. The speed of the observer is $v$. We assume that the path of the observer can be approximated by a straight line over distances of the order of the communication range of a sensor. We also assume that the observer is not power-constrained.

The sensors are all identical. Every sensor has the same kind of battery, uses the same communication range $R_{\max }$ and uses the same data rate to transmit 
information to the observer when it is within range. The rate at which sensors collect data about the phenomenon being monitored is also same for all nodes.

Each sensor needs time $T$ to send all its data to the observer. The observer must be within range for the entire interval $T$, for if the observer moved out of range before $T$, communication would be unsuccessful resulting in a failure that we call outage. Communication takes place at constant rate and over a continuous time interval $T$. Communication between sensors and the observer is always single-user and over a single hop. There is no relaying, multiple-access or multicasting.

For single hop communication, the observer should come within communicable range of every sensor inside the given area $A$ from some point on its fixed path. Let $R$ be the distance for which every sensor node lies within a distance $R$ from some point on the path of the observer. Note that $R$ and the range $R_{\max }$ are different. Every sensor node comes within a distance $R$ from the path of the observer. But for successful communication it is not simply enough that the observer should come within $R_{\max }$ of the sensor. It must remain within range for a period of $T$ or more. The communication range $R_{\max }$ is chosen to satisfy

$$
R_{\max } \geq \sqrt{R^{2}+(v T / 2)^{2}} .
$$

in order to ensure that every node remains within range for at least $T$ seconds (this uses the straight-line path assumption). The relation between $R$ and $R_{\max }$ is explained in Fig. 2.

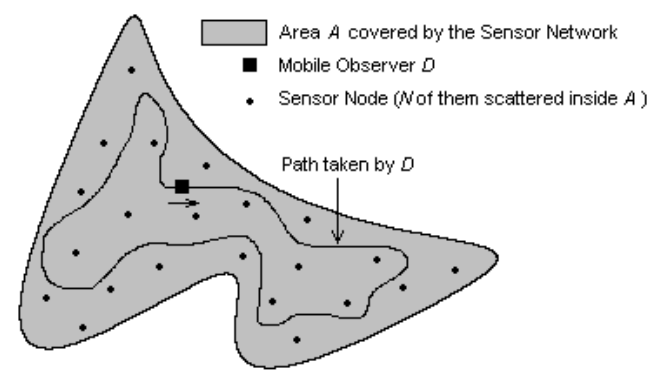

Fig. 1. Diagram of the Sensor Network

\section{Process of Data Collection}

As mentioned earlier, two node distribution models are studied in this paper. In the first model, nodes are assumed to be independently and uniformly scattered over the area $A$. In the second model, we assume that sensors are randomly distributed subject to the constraint that no two sensors are less than $d$ meters apart. 


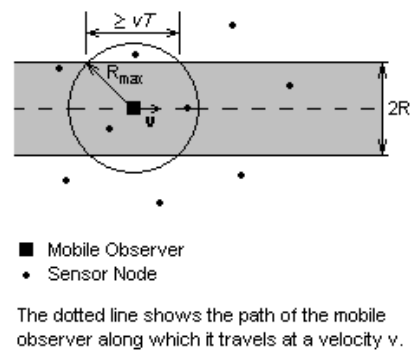

Fig. 2. Relation between $R$ and $R_{\max }$

In the first model, we shall analyze the tradeoff between communication power and expected outage during data collection using a queuing formulation. In the second model, we shall show that it is possible to guarantee zero outage if the sensor separation is above a threshold.

\subsection{Independently and Uniformly Distributed Nodes}

Sensors are independently and uniformly scattered over the given area. Once they have been scattered, their locations are fixed. Thus, the distribution that we are referring to is the a priori distribution of sensors. The results we will obtain from the analysis below will indicate average performance over all possible sets of node positions.

Under this distribution, it may not be possible to collect data from all sensors. This is because random scattering introduces the possibility of several nodes being located close together, all trying to send data and the observer not being able to receive data from all of them. The presence of redundant nodes alleviates this problem to some extent. However, performance does get affected when the fraction of nodes unable to transmit is above a certain threshold. The fraction of nodes in outage may be reduced below this threshold by either increasing the transmission range $R_{\max }$ or by increasing the data rate. Note that increasing the transmission range and the data rate both involve increasing transmission power. The important questions to answer in this context are:

1. What is the minimum power with which we can collect data from the specified fraction of nodes?

2. What combination of $R_{\max }$ and $T$ achieves this?

As the observer moves, new sensors come within range and ones that were within range go out of range. We now provide a suitable mathematical formulation for this process.

In a time interval of length $t$, the observer moves a distance $v t$. Nodes in an area $2 R v t$, which were previously out of range, come within range. The observer may be busy when a new node comes within range, in which case, the node has to wait in order to send its data. If the observer is not busy, the sensor can start sending its data immediately. Once the observer has started communicating with this node, it will not listen to any other node that may come within range. This is 
analogous to a queue where sensors arrive according to a certain random process and the observer listens to them one by one.

An important point is that it is futile to start communicating with a sensor node that will not stay within range long enough. For each sensor there is a maximum waiting time, which is a function of its distance from the path of the observer. If the observer does not start communicating with the node before this time, it will be impossible for the sensor to send all its data, resulting in outage. This is depicted in Fig. 3.

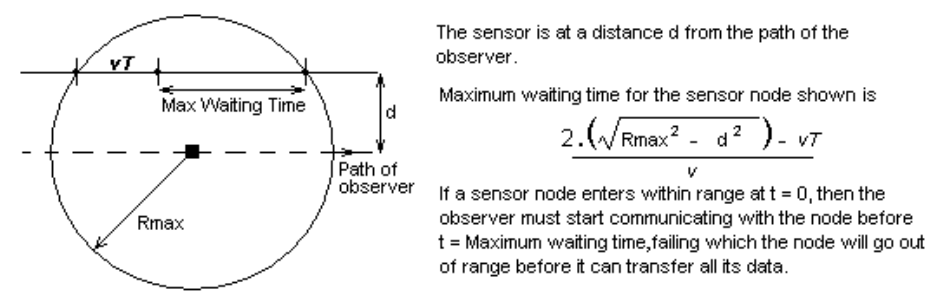

Fig. 3. Relation between Waiting Time and Outage

If $N$ nodes are independently and uniformly distributed over area $A$, then the interarrival times may be modeled using the following $p d f$ (see Appendix A)

$$
p_{\text {arrival }}(t)=\frac{2 R v N(A-2 R v t)^{N-1}}{A^{N}} ; \quad 0 \leq t \leq \frac{A}{2 R v}
$$

Also, the distance $d$ shown in Fig. 3 is uniformly distributed from 0 to $R$ in magnitude as a consequence of the independent and uniform distribution of nodes. Based on this, the $p d f$ of waiting times (see Appendix B) is

$p_{w}(W)=\frac{v^{2}(W+T)}{4 R \sqrt{R_{\max }^{2}-v^{2}(W+T)^{2} / 4}} ; \frac{2 \sqrt{R_{\max }^{2}-R^{2}}}{v}-T \leq W \leq \frac{2 R_{\max }}{v}-T$

A cycle, during which the observer moves through the entire area $A$ once, can be simulated like a queue using these two distributions. For purposes of the simulation, it has been assumed that the observer has exact knowledge of the waiting time of each sensor that comes within range (as if aided by an oracle), which allows it to perform optimal scheduling. In practice, a protocol may be designed to ascertain the waiting times through exchange of information between the sensor and the observer (see Sect. 5).

Numerical Results. Increasing the value of $R_{\max }$ reduces outage and keeps the observer busy more often. When we increase the range, we increase the waiting times. As a consequence, sensors can wait longer for their turn before they finally go out of range resulting in outage. It is as if we have increased the size of the input buffer of a queue to smooth out burstiness in the input. 
This improvement in performance comes at a price. Increasing the communication range while keeping the data rate same involves spending more power. For doubling $R_{\max }$, while keeping the worst case $S N R$ same, one needs to increase transmission power at least four times (actually $2^{\gamma}$ times where $\gamma$ is the path attenuation constant).

Performance is also seen to improve when $T$ is reduced while keeping $R_{\max }$ fixed. As in the case of increasing $R_{\max }$, decreasing $T$ also involves more power.

Fig. 4 and Fig. 5 show the pattern of changes in outage and the percent of time the observer is receiving information with changes in $T$ and $R_{\max }$ respectively. These observations suggest that there may be multiple combinations of the parameters $R_{\max }$ and $T$ that can be used to achieve outage below a specified limit. The designer must pick the one that consumes minimum energy in order to maximize network life.

We chalk out the procedure for power-optimization with the aid of an example. Table 1 contains a list of the system parameters used for simulations.

Table 1. List of System Parameters

\begin{tabular}{rr}
\hline Parameter & Value \\
\hline$\gamma=$ Path attenuation of wireless channel & $R$ \\
$A=$ Area covered by the sensor network & $100 \mathrm{sq} \mathrm{km}$ \\
$v=$ Observer velocity & $15 \mathrm{~m} / \mathrm{sec}$ \\
$N=$ Number of nodes & 3000 \\
$R_{\text {sens }}=$ Rate at which a sensor collects data & $160 \mathrm{bps}$ \\
$B=$ Bandwidth of the system & $100 \mathrm{kHz}$ \\
$N_{0}=$ Noise power spectral density & $10^{-19} \mathrm{Watt} / \mathrm{Hz}$ \\
$T_{\text {cycle }}=$ Time needed to complete one cycle & $\sim A /(2 R v)$ \\
$D_{\text {cycle }}=$ Data collected by a node in one cycle & $R_{\text {sens }} * T_{\text {cycle }}$ \\
\hline
\end{tabular}

For different values of $T$, the minimum value of $R_{\max }$ is found (through simulation) for which the expected outage is not in excess of the specified $20 \%$. The power $P$ is calculated using Shannon's expression for channel capacity and equating it to the data rate $D_{\text {cycle }} / T$, i.e.,

$$
P=B N_{0} R^{\gamma}\left(2^{D_{\text {cycle }} / T B}-1\right),
$$

and the energy consumed by a node per cycle for the purpose of transmission is calculated by multiplying this power by the time $T$. The power and energy obtained for different $T$ are plotted in Fig. 6 from which it can be seen that there exists a certain point where the energy consumed is minimized. Fig. 7 shows the different values of $R_{\max }$ that were found to ensure less than $20 \%$ outage. It is important to note that the energy spent varies significantly depending on the 


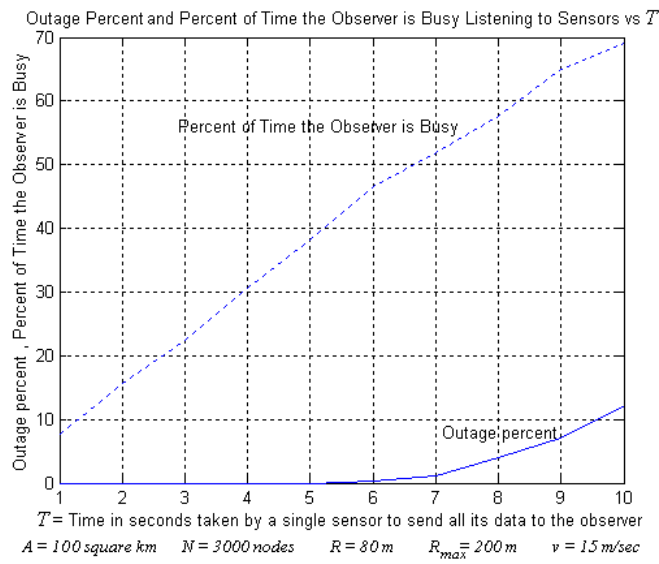

Fig. 4. Effect of $T$ on performance

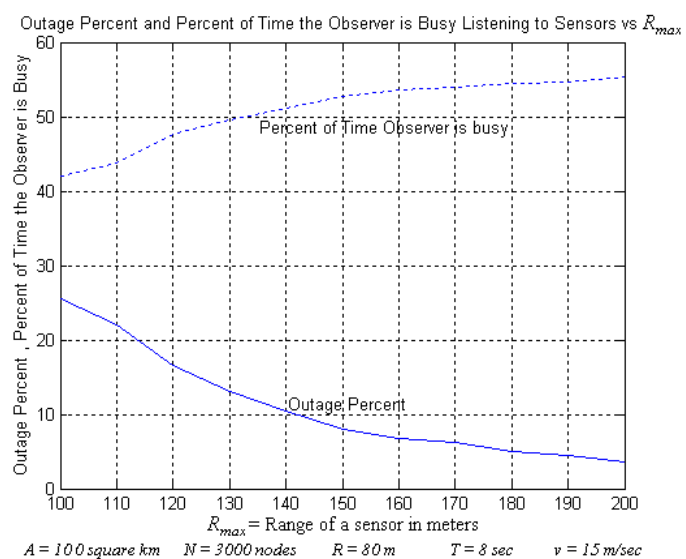

Fig. 5. Effect of $R_{\max }$ on performance

choices of $T$ and $R_{\max }$, thus reiterating the fact that a suitable choice of the system parameters is crucial for power efficiency.

Comment on the Choice of Velocity. We have carried out all our analysis assuming that the observer moves at a fixed speed $v$. In practice, the velocity of the observer has a certain probability distribution and the outage that we measure depends on this distribution. Moreover, this distribution may not be stationary. The question is: which velocity should we consider as $v$ for designing the system so that the specified maximum outage is not exceeded?

In most scenarios, we would recommend either the maximum speed, or a speed that is very rarely exceeded, as the choice for $v$. Note here that the worst outage results when the observer is moving fast, not when it is static or moving 


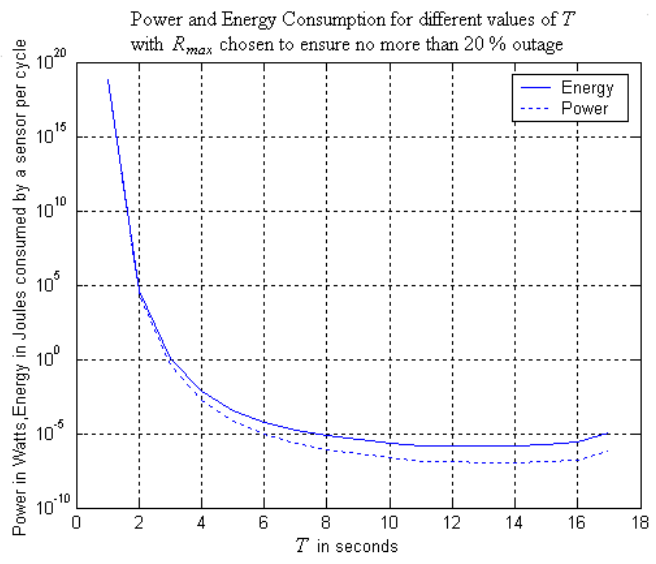

Fig. 6. Power and Energy Optimization

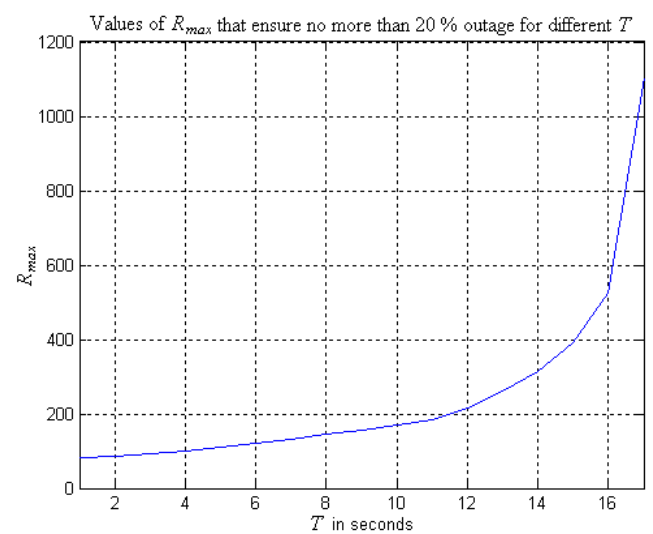

Fig. 7. Values of $R_{\max }$ to ensure outage $<20 \%$

slowly. Choosing the average speed as $v$ could be potentially harmful due to the non-stationary nature of the distribution of velocities. For example, the average speed of a bus could be $30 \mathrm{mph}$, but the bus might consistently travel at $40 \mathrm{mph}$ on some roads, so that if $v$ were chosen to be $30 \mathrm{mph}$, it would result in high outage near those roads thereby impairing the ability of the sensor network to gather data from there.

\subsection{Randomly Distributed Nodes with Minimum Separation Constraint}

In sensor networks where nodes are randomly distributed subject to the constraint that no two nodes can be closer than $d$ (minimum separation), zero outage can be guaranteed by an appropriate choice of parameters. This is signif- 
icant because outage is not acceptable in many applications and yet unavoidable if we allow the nodes to be randomly scattered without additional constraints.

It can be shown that the condition

$$
d \geq \sqrt{(2 R)^{2}+(v T)^{2}} .
$$

is sufficient for guaranteeing zero outage (see Appendix C). This condition ensures that the time gap between one sensor entering within the range of the observer and the next sensor is at least $T$. This condition can be met by reducing $T$ if the sensors are positioned to satisfy

$$
d>2 R \text {. }
$$

\section{Power Comparison with Sensor Networks Having Static Observers}

To quantify the power savings afforded by observer mobility, we compare our sensor network model with static sensor network models covering an area $A$ that is circular in shape with one observer at the center of the circle. The sensor network has $N$ sensors in all cases. These sensors are uniformly distributed over the entire area. The three cases that we compare are shown in Fig. 8. Case 1 corresponds to our proposed model. In case 2 , the observer is static and each sensor node transmits its data directly to the observer over a single hop. In case 3 , each sensor node sends its data to the observer over multiple hops.
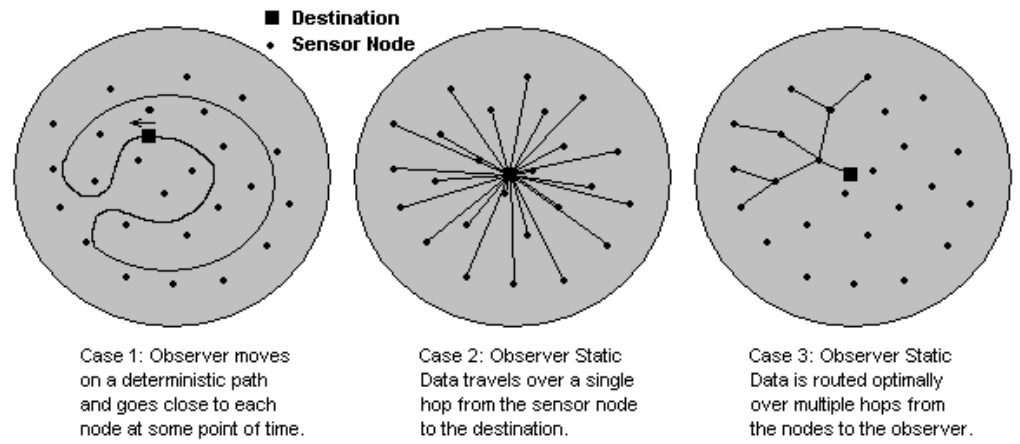

Fig. 8. Sensor Network Models

For purposes of comparison, we consider an area of $100 \mathrm{sq} \mathrm{km}$. Each sensor measures data at a rate of $1 \mathrm{kbps}$. The remaining parameters have values provided in Table 1 unless mentioned otherwise.

\subsection{Case 1: Mobile Observer}

We choose $R=500 \mathrm{~m}$ (this seems to be a reasonable distance for citywide public transport systems). The communication bandwidth available is $1 \mathrm{MHz}$. In the 
proposed model, the energy consumed by a single sensor per cycle was found to be around 0.3 microjoule using the technique outlined in section 3.1. In general (see Table 1),

$$
\text { Energy consumed per sensor }=T B N_{0} R^{\gamma}\left(2^{D_{\text {cycle }} / T B}-1\right) \text { Joule } / \text { cycle . }
$$

\subsection{Case 2: Static Observer - Single Hop Communication}

In this case, different sensors need to have different communication ranges in order to send their data to the observer. We assume that each sensor transmits at the same power. As a result, the sensors that are farther away from the observer will be able to transmit at a slower data rate and their communication will take more time. We would like to find how much power $P$ each sensor needs to have so that the rate at which the network collects data can be matched by the rate at which data is communicated to the observer. For fair comparison, we assume that $20 \%$ nodes do not send anything as in case 1 . Since a node at a distance $r$ from the observer transmits at a data rate of

$$
D(r)=B \log _{2}\left(1+\frac{P}{B N_{0} r^{\gamma}}\right) b p s
$$

over a cycle period of $T_{\text {cycle }}$, the node collects $D_{\text {cycle }}$ bits of data (see Table 1 ). The time taken to communicate this to the observer is $D_{\text {cycle }} / D(r)$. This quantity, summed over all nodes should equal $T_{\text {cycle }}$. The value of $P$, for which this equality occurs, is the transmission power required by every node. This value of $P$ was calculated to be about 6.44 microwatts (for uniformly distributed sensors) from which the average energy spent per node per cycle was calculated to be 111.8 microjoules. This is over 300 times the average energy consumption per node per cycle in case 1 .

\subsection{Case 3: Static Observer - Multi Hop Communication}

The power consumption in this case is difficult to calculate. It depends on the actual routes chosen for communication between different nodes and the sensor, which in turn depend on the locations of nodes. To simplify the situation, we consider an optimistic scenario where sensors are located in a uniform triangular lattice as shown in Fig. 9. As earlier, here also, for the sake of fair comparison, we assume that $20 \%$ of the nodes do not send data to the observer.

If $N$ nodes were placed in this fashion over an area $A$, then the separation between neighboring nodes would be approximately

$$
d=\sqrt{2 A / \sqrt{3} N} .
$$

In such a situation, a node, which is at a distance $r$ from the observer, would have to send its data over at least $\lceil r / d\rceil$ hops, each of length $d$. The value of $d$ is calculated to be $196.2 \mathrm{~m}$ in our case. 


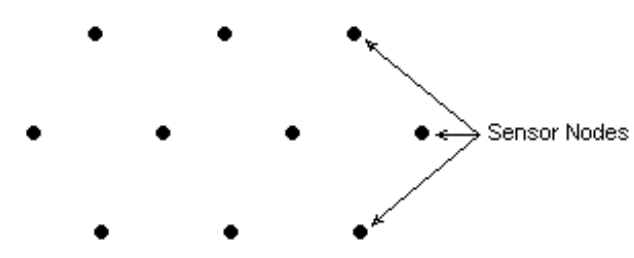

Fig. 9. Arrangement where sensors lie on the vertices of equilateral triangles

Over a cycle period of $T_{\text {cycle }}$, a node at a distance $r$ from the observer collects $D_{\text {cycle }}$ bits of data (see Table 1$)$. This data has to travel over $\lceil r / d\rceil$ hops, each of length $d$. Here again, we assume that the nodes are identical, they use the same transmission power and have a transmission range of $d$ (they need no more). Since, we have also assumed that the observer can receive only from one sensor at a time, it follows that a single link should be able to transmit at the rate at which the network collects information. From this it follows that the transmission power over a single hop will be

$$
P^{\prime}=B N_{0} d^{\gamma}\left(2^{0.8 N R_{\text {sens }} / B}-1\right)
$$

Over a cycle period of $T_{\text {cycle }}$, a single node collects $D_{\text {cycle }}$ bits of data (see Table 1). If this information is transmitted at a rate of $0.8 N R_{\text {sens }}$, the time taken will be $D_{\text {cycle }} /\left(0.8 N R_{\text {sens }}\right)=T_{\text {cycle }} /(0.8 N)$ secs. The energy consumed to send a single sensor's information over a single hop per cycle is then $T_{\text {cycle }} P^{\prime} /(0.8 N)$ Joule. Multiplying this by the average number of hops over which data travels gives us the average energy consumed per sensor. The average number of hops is almost equal to $(2 \sqrt{A}) /(3 \sqrt{\pi} d)$. Thus, on average, $\left(2 \sqrt{A} T_{\text {cycle }} P^{\prime}\right) /(2.4 \sqrt{\pi} N d)$ Joule will be expended per sensor per cycle. For our system, this is $0.88 \mathrm{mi}-$ crojoule, which is about thrice the energy expended by a sensor per cycle in case 1 .

\section{Communication Protocol}

The protocol for communication between nodes and the observer needs to be designed in such a way that individual sensors have very little responsibility apart from that of collecting data and communicating when requested. Medium access control, resolving contention, dealing with collisions and various kinds of failures will be handled by the observer since, by our assumption, it is not power constrained.

The life of a sensor network may be loosely divided into three phases- startup, steady state and failure [11]. We will describe briefly, the tasks to be performed by the protocol at each stage.

\subsection{Startup Phase}

Sensor nodes boot up individually. Each sensor has a unique address. At this time, the observer knows nothing about the positions of individual sensors. Nei- 
ther do sensors know anything about the path of the observer. During the startup phase, the observer and the sensors exchange information that helps them to acquire such knowledge about each other. The startup phase consists of two different cycles.

Cycle 1. Sensors listen to the channel periodically at quick intervals to check if there is an observer within range. The observer, in this phase, goes on its regular path while continuously broadcasting a beacon signal. The strength of this beacon is same as the strength at which sensor nodes will typically transmit in the steady phase. Each sensor is able to measure:

1. How often the observer comes within range?

2. How long it stays within range?

Wireless channels are time varying and to obtain reliable estimates of these parameters, this cycle should be repeated a stipulated number of times.

Cycle 2. Here, the observer travels on its regular path broadcasting a beacon. When a sensor hears the observer, it responds with an RTS (Request To Send) packet containing its address. A collision-resolution strategy, based on random backoff (similar to 802.11), is used to resolve collisions among RTS packets sent by different sensors. When the observer hears an RTS from a particular sensor, it stops broadcasting the beacon, sends a CTS (Clear To Send) addressed to the sensor, and then the sensor node sends a small packet, which contains information about the parameters measured in cycle 1. This information will help the observer to decide priority when there are multiple sensor nodes in range waiting to transfer data (during the steady phase). The observer sends an ACK (ACKnowledgement) to the sensor node after it has received this packet.

Since there is the possibility of packet loss, this cycle should also be repeated several times. When cycle 2 is repeated, the only sensors that respond to the observer's beacon are those that have not received an ACK. The information exchanged in this cycle is crucial for efficient steady phase operation and all sensors should be able to send their packets to the observer.

\subsection{Steady Phase}

The observer has accurate knowledge about the positions of different sensor nodes. Using this information, and using the knowledge of its own position, the observer initiates communication, using a wake signal, with sensors that it knows to be within range. When there are several sensors within range, the observer initiates communication with that node which will go out of range first. In a sense, the observer assigns higher priority to sensors that can wait less.

In the steady phase, sensor nodes predict when the observer is likely to come close based on the information gathered during startup. They monitor the channel only when the observer is expected to be nearby. In [6], it has been 
indicated that the power consumed in channel monitoring can be a major chunk of the total power consumption of a sensor node. By reducing channel monitoring time, the life of a sensor node is increased significantly.

This protocol should perform well for sensor networks with static nodes. If the nodes are mobile and move by distances of the order of the separation between sensors or more in a relatively short time period, then it is difficult to do efficient scheduling. In certain cases, it may help to keep updating the information collected during the startup phase every cycle or once in every few cycles.

\subsection{Failure Detection}

The observer can detect node failures through their consistent inability to respond to wake calls and suitably reschedule the remaining nodes. When a significant number of nodes have failed, the network will no longer be able to gather sufficient data.

\section{Conclusions}

We showed that predictable mobility can be used to significantly reduce communication power in sensor networks. There are several advantages that predictability has over random mobility. One of them is boundedness of the transmission delay. Determinism of the path also makes it possible to predict the point where the observer and the mobile should communicate to save maximum power.

Much work on exploiting mobility remains to be done. Our future work will focus on, among other issues, combining relaying with predictable mobility to yield even higher power savings over multi-hop static sensor networks. For applications with loose performance requirements, random mobility can also be exploited.

\section{References}

1. Min, R., Chandrakasan, A.: Energy-efficient communication for ad-hoc wireless sensor networks. In: Conference Record of the Thirty-Fifth Asilomar Conference on Signals, Systems and Computers. Volume 1. (2001) 139-143

2. Pottie, G.J.: Wireless sensor networks. In: Information Theory Workshop. (1998) 139-140

3. Salhieh, A., Weinmann, J., Kochha, M., Schwiebert, L.: Power efficient topologies for wireless sensor networks. In: International Conference on Parallel Processing. (2001) 156-163

4. Min, R., Bhardwaj, M., Cho, S.H., Sinha, A., Shih, E., Wang, A., Chandrakasan, A.: An architecture for a power-aware distributed microsensor node. In: IEEE Workshop on Signal Processing Systems. (2000) 581-590

5. Estrin, D.: Wireless sensor networks: Application driver for low power distributed systems. In: International Symposium on Low Power Electronics and Design. (2001) 194 
6. Rabaey, J.M., Ammer, J., Karalar, T., Li, S., Otis, B., Sheets, M., Tuan, T.: Picoradios for wireless sensor networks: the next challenge in ultra-low power design. In: Digest of Technical Papers. ISSCC. IEEE International. Volume 2. (2002) 156-445

7. Liu, C.H., Asada, H.: A source coding and modulation method for power saving and interference reduction in ds-cdma sensor network systems. In: Proceedings of the American Control Conference. Volume 4. (2002) 3003-3008

8. Pei, G., Chien, C.: Low power tdma in large wireless sensor networks. In: Military Communications Conference. Volume 1. (2001) 347-351

9. Chien, C., Elgorriaga, I., McConaghy, C.: Low-power direct-sequence spreadspectrum modem architecture for distributed wireless sensor networks. In: International Symposium on Low Power Electronics and Design. (2001) 251-254

10. Grossglauser, M., Tse, D.: Mobility increases the capacity of ad hoc wireless networks. In: INFOCOM. Proceedings. IEEE. Volume 3. (2001) 1360-1369

11. Sohrabi, K., Gao, J., Ailawadhi, V., Pottie, G.J.: Protocols for self-organization of a wireless sensor network. IEEE Personal Communications 7 (2000) 16-27

\section{A Distribution of Interarrival Times}

The $p d f$ of interarrival times may be derived as follows.

In a time period $t$, the observer travels a distance $v t$. Nodes within an area $2 R v t$ that were previously out of range now come within range. If $p(t)$ were the interarrival pdf then ${ }^{1}$ :

$\int_{0}^{t} p(x) d x$

$=$ Probability that at least one node enters within range in time $t$

$=1-($ Probability that no node enters within range in time $t)$

$=1-\left(\frac{A-2 R v t}{A}\right)^{N}$

Taking the derivative on both sides with respect to $t$ yields Eqn. 2, which is the $p d f$ of interarrival times.

\section{B Distribution of Waiting Times}

The $p d f$ of maximum waiting times may be derived as follows. The concept of maximum waiting time is explained in Fig. 3. The maximum waiting time for a sensor node that is at a distance $r$ from the path of the observer is

$$
W(r)=\left(2 \sqrt{R_{\max }^{2}-r^{2}}-v T\right) / v .
$$

Note that $r$ is uniformly distributed from 0 to $R$. Hence the $p d f$ of waiting times may be obtained by transforming this uniform $p d f$

$$
p_{w}(W)=\frac{p_{r}(r)}{\left|\frac{d W(r)}{d r}\right|}
$$

which yields the $p d f$ given in Eqn. 3 .

\footnotetext{
${ }^{1}$ This follows from the fact that nodes are independently and uniformly distributed and the probability for any single node to be outside the area $2 R v t$ equals $(A-$ $2 R v t) / A$. Note that the time $t$ cannot exceed $(A / 2 R v)$ because in this time the observer's range covers an area $A$ i.e. the entire area.
} 


\section{Condition for Guaranteeing Zero Outage}

The sufficient condition for ensuring zero outage with minimum separation of nodes $d$ is derived as follows( see Fig. 10(a)). Data transfer from a node to the observer takes time $T$. If we can ensure that the entry of two nodes into the range of the observer is spaced in time by $T$, then outage will not occur. Thus, if a sensor node is placed anywhere on the arc $A B$ (see Fig. 10(a)), then no other node can lie within the shaded region $A B C D$. This implies that the minimum separation $d$ must be greater than or equal to the distance from any point on arc $A B$ to any other point on the boundary of $A B C D$ i.e.

$$
d \geq \max (|x-y|) ; \quad x \in A B, y \in A B C D .
$$

Let $x=U$ and $y=V$ be the pair for which $|x-y|$ is maximized.

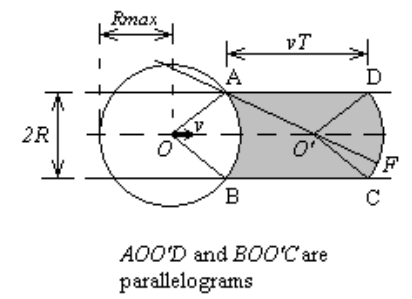

The observer moves along the line $O O^{\prime}$ with a velocity $v$. The circle denotes its range.

(a)

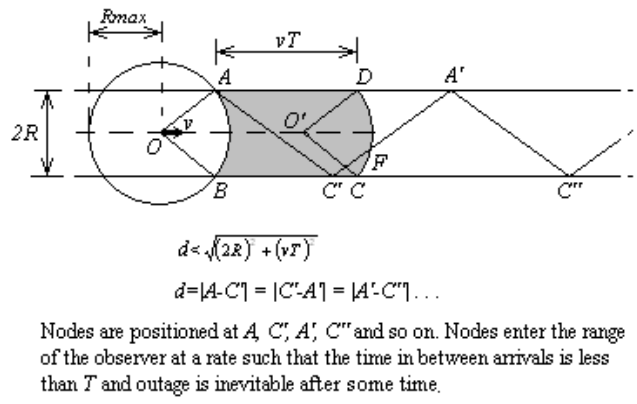

(b)

Fig. 10.

Proposition: $U=A$ and $V=C$ (or $U=B$ and $V=D$ ) are the pair that maximizes $|x-y|$. This is proved as follows:

Claim 1: Choose and fix an arbitrary point $x=P \in A B$. Then, the point $y=Q \in A B C D$, for which $|P-y|$ is maximized does not lie on the line segments $B C$ or $A D$ except possibly that $Q$ is one of the end points $A, B, C$ or $D$.

Proof: Assume, for a contradiction that $Q \in B C Q \neq B, C$ (similar proof if $Q \in A D)$. Then $\max (\angle P Q B, \angle P Q C) \geq 90^{\circ}$. Thus, $\max (|P-B|,|P-C|)>$ $|P-Q|$. Hence, we have a contradiction to the fact that $y=Q$ maximizes $|P-y|$.

Corollary 1: Since this claim holds for arbitrary $x$, it must hold for $x=U$, the point which achieves the overall maximum. Therefore, $V \in\{A B, C D\}$.

Claim 2: Choose and fix an arbitrary point $x=P \in A B$. Suppose that the point $y=Q \in A B C D$ is the point for which $|P-y|$ is maximized. Then, unless $P$ is one of the end points of $A B,|P-Q|<|U-V|$. In other words, $U \in\{A, B\}$. 
Proof: This is similar to the previous proof. Since $\max (\angle Q P B, \angle Q P A)>90^{\circ}$, therefore $\max (|A-Q|,|B-Q|)>|P-Q|$ which means $|P-Q|<|U-V|$.

Corollary 2: $V$ lies on $C D$ for if $V$ were to lie on $A B$, by moving horizontally to the corresponding point on $C D$, one could show that this point is farther from $U$ than $V$ is.

The problem has therefore reduced to that of finding $V$ from the set of points on $C D$. $U$ has been ascertained to be either $A$ or $B$ (which one we choose makes no difference). Consider the line passing through $A$ and $O^{\prime}$. This line cuts the $\operatorname{arc} C D$ if and only if

$$
(2 R)^{2}+(v T)^{2}>\left(2 R_{\max }^{2}\right)
$$

which is in direct contradiction to Eqn. 1. The case of interest is when the line passing through $A$ and $O^{\prime}$ does not cut $C D$. This happens when $A$ is a point that lies within the circle centered at $O^{\prime}$ and having radius $R_{\max }$. We propose the following theorem for this situation.

Theorem: When the line passing through $A$ and $O^{\prime}$ does not cut $C D$,

$$
|A-C|=\max (|x-y|) ; \quad x \in A B, y \in A B C D .
$$

Proof: If $A$ were to lie on the circumference of the circle centered at $O^{\prime}$ having radius $R_{\max }$, and $F$ were an arbitrary point on $\operatorname{arc} C D$, then $\angle A F C$ would have been $90^{\circ}$. Now as $A$ is moved along a straight line towards $D$, so that it enters within the circle, $\angle A F C$ increases monotonically. The important point is that $\angle A F C>90^{\circ}$. As a consequence, $|A-C|>|A-F|$ for all choices of $F$.

This is the result using which we obtain a meaningful relationship between the minimum separation $d$ and our system parameters. Since

$$
|A-C|=|B-D|=\sqrt{(2 R)^{2}+(v T)^{2}},
$$

we conclude that

$$
d \geq \sqrt{(2 R)^{2}+(v T)^{2}}
$$

is a sufficient condition to guarantee zero outage.

An interesting point to note is that the above condition is also necessary to guarantee zero outage, meaning that if this condition is not satisfied, then it is possible to arrange sensors in a bad way, so that outage occurs. Fig. 10(b) shows one such arrangement of sensors where outage is unavoidable with

$$
d<\sqrt{(2 R)^{2}+(v T)^{2}} .
$$

